\title{
Caracterización morfológica de seis variedades parentales de yacón (Smallanthus sonchifolius) y trece cruzas obtenidas de un plan de hibridación
}

\section{Morphologic characterization of six parental varieties of yacón (Smallanthus sonchifolius) and thirteen crosses obtained from a hybridization plan}

\section{Diana P. Vegas Albino ${ }^{1 *}$, Olga Bracamonte Guevara ${ }^{1}$ y Andrés Valladolid Cavero ${ }^{2}$}

1 Laboratorio de Citogenética. Facultad de Ciencias Biológicas, Universidad Nacional Mayor de San Marcos, Lima, Perú. Casilla 11-058, Lima 11, Perú. Tel.: +51 6197000 - 1529; fax: +51 6197000 - 1509 .

2 Andean Roots SRL. Carretera Central Huánuco-Lima, km. 40, Ambo, Huánuco, Perú.

* Autor para correspondencia

Email Diana P. Vegas: diana_vegas_albino@hotmail.com

Email Olga Bracamonte: obracamonteg@hotmail.com

Email Andrés Valladolid: a.valladolid@andeanroots.com.pe

\begin{abstract}
Resumen
En el presente trabajo se caracterizan morfológicamente los parentales y las plantas obtenidas a partir de los cruzamientos dialélicos de yacón (Smallanthus sonchifolius), con la finalidad de identificar la diversidad obtenida y confirmar la naturaleza híbrida de estas. Tres descriptores morfológicos de raíz fueron usados para caracterizar a los parentales y a 68 de las 162 plantas obtenidas de las cruzas, cada una con sus respectivas replicaciones, en total se evaluaron 834 plantas. Finalmente se determinaron 44 híbridos, 8 plantas con hibridación no determinada al no identificar si existió cruza, pero que heredaron solo los caracteres del progenitor femenino o son replicaciones de este, 9 plantas con un origen híbrido no específico al no poder determinarse si existió hibridación heredando caracteres compartidos por ambos progenitores o solo caracteres del progenitor femenino o no, 7 plantas cuyo progenitor masculino no es el correspondiente a la cruza, 2 replicaciones de 3 híbridos y 1 replicación de 1 de las 9 plantas con un origen híbrido no específico también presentan un progenitor masculino diferente al de su cruza. Se observó precocidad del parental V24 y pérdida del carácter de moteaduras irregulares púrpura rojizo (indicador de antocianinas) en las 44 plantas con parentales que presentaban este carácter.
\end{abstract}

Palabras clave: Hibrido; caracterización morfológica; germoplasma; diversidad genética; descriptor.

\section{Abstract}

The aim of this study is to characterize morphologically the parental and plants and those obtained from the diallel crosses, to identify the variety obtained and to confirm the hybrid nature of these. Three morphological descriptors of root were used to characterize the parental and 68 of the 162 plants obtained from crosses, each with their replication, in total 834 plants were evaluated. Finally 44 hybrids were obtained, 8 plants with no specific hybridization to no identify if there was cross but inheriting only the characters of the female parent or are replications of this, 9 plants with a non-specific hybrid origin unable to determine whether there was hybridization inheriting shared characters by both parents or single characters of the female parent or not; 7 plants whose male parent is not corresponding to the cross, 2 replications of three hybrids and one replication 1 of 1 of 9 plants with a non-specific hybrid origin also presented a different male parent. The parental V24 showed precocity and the loss of irregular reddish purple specks (anthocyanins indicator) was observed in 44 plants with parental that has this character.

Keywords: Hybrid; morphological characterization; germplasm; genetic diversity; descriptor.

Citación:

Vegas Albino D.P., O. Bracamonte Guevara \& A. Valladolid Cavero. 2015. Caracterización Morfológica de seis variedades parentales de yacón (Smallanthus sonchifolius) y trece cruzas obtenidas de un plan de hibridación. Revista peruana de biología 22(2): 175 - 192 (Octubre 2015). doi: http://dx.doi.org/10.15381/rpb.v22i2.11352

$\begin{array}{ll}\text { Presentado: } & 22 / 05 / 2015 \\ \text { Aceptado: } & 26 / 08 / 2015\end{array}$

Aceptado: $\quad 26 / 08 / 2015$

Publicado online: 14/10/2015
Información sobre los autores:

DPVA, OBG, AVC: realizaron el diseño experimental; DPVA AVC: realizaron los experimentos; DPVA: analizó los datos; DPVA: redactó el manuscrito; DPVA, OBG, AVC: revisaron y aprobaron el manuscrito. Los autores no incurren en conflictos de intereses.

Fuentes de financiamiento: Esta investigación pudo ser realizada con el financiamiento de FIDECOM (Fondo de Investigación y Desarrollo para la Competitividad) del Ministerio de la Producción del Perú y de la empresa Andean Roots SRL. 


\section{Introducción}

El yacón (Smallanthus sonchifolius (Poepp.) H. Rob.) es un cultivo importante que se desarrolla desde el sur de Colombia hasta el norte de Argentina, entre los 1800 y $2800 \mathrm{~m}$ de altitud (Svobodová et al. 2013), en las últimas décadas la producción de yacón en la región andina se ha incrementado debido a la demanda por las presuntas propiedades médicas tanto de raíces y hojas, y sus cultivos se han sido expandidos a varios países como Nueva Zelanda, Japón y Brasil (Campos et al. 2012).

La raíz del yacón tiene características particulares que la convierten en importante. Entre el 83 y $90 \%$ del peso fresco de la raíz es agua; mientras que los carbohidratos representan alrededor del $90 \%$ del peso seco de las raíces recién cosechadas, y de los cuales entre el 50 y $70 \%$ son fructooligosacáridos (v.g. inulina); el resto de carbohidratos lo conforman la sacarosa, fructosa y glucosa. Sin embargo, la composición relativa de los diferentes azúcares varía significativamente debido a diferentes factores como el cultivo, la época de siembra y cosecha, tiempo y temperatura en poscosecha, entre otros (Coronado, 2013). Los fructooligosacáridos han sido asociados con efectos benéficos como: reducir el colesterol LDL, prevenir y tratar el estreñimiento, reducir el riesgo de cáncer de colon, restaurar la microflora benéfica del colon y bajar de peso a personas obesas, entre otras que han convertido al yacón en un recurso importante en el mercado de los productos dietéticos y las personas que padecen diabetes (Seminario et al. 2003).

La propagación del yacón es eminentemente clonal (Soto 2012). La escasa reproducción sexual ha sido atribuida principalmente al origen alopoliploide del yacón, lo que condiciona la formación de gametos desbalanceados e inviables durante la meiosis, lo cual se relaciona a la alta esterilidad de los granos de polen y a la escasa o nula viabilidad de las semillas (Grau \& Rea 1997). La baja capacidad de reproducción sexual es una limitación importante para el mejoramiento genético del yacón (Viehmannova et al. 2013). Además, Grau y Rea (1997) afirman que el yacón es una planta de polinización cruzada, ya que se ha observado que la apertura y receptividad de las flores femeninas en los capítulos se da antes que las flores masculinas liberen el polen, necesitando de agentes polinizadores para que se realice la fecundación. La polinización por insectos es aparentemente favorecida porque se ha encontrado que el grano de polen es viscoso y posee acúleos en su superficie. También, el prolongado inicio de la floración y la asincrónica formación de los capítulos en la planta favorecerían una polinización cruzada (Mansilla et al. 2010). A pesar de estas características de reproducción se ha podido observar cierta variabilidad (Milella et al. 2005, Mansilla et al. 2006, Svobodová et al. 2013).

La descripción varietal es un conjunto de observaciones que permiten distinguir y caracterizar a una población de plantas que constituyen una variedad (Laguna, et al., 2006). Un descriptor es una característica o atributo cuya expresión es fácil de medir, registrar o evaluar y que hace referencia a la forma, estructura o comportamiento de una accesión. Los descriptores de caracterización permiten la discriminación fácilmente entre fenotipos. Generalmente son caracteres altamente heredables que pueden ser detectados a simple vista y se expresan igualmente en todos los ambientes (Franco \& Hidalgo, 2003).

En los meses de febrero y marzo del 2012, Manrique et al. (2014) realizaron cruzamientos entre seis accesiones de yacón, provenientes del banco de germoplasma del Instituto Nacional de Innovación Agraria (INIA), mediante un diseño de cruzas dialélicas directas y recíprocas, con el objetivo de analizar la diversidad genética a nivel molecular mediante marcadores Random Amplified Polymorphic DNA (RAPDs). A partir de estos cruzamientos se obtuvieron 162 semillas viables, las cuales germinaron y produjeron plantas adultas. El objetivo del presente trabajo es caracterizar morfológicamente los parentales y las plantas obtenidas a partir de los cruzamientos dialélicos para identificar la diversidad obtenida y para confirmar la naturaleza híbrida de estas.

\section{Materiales y métodos}

Material vegetal.- Seis accesiones de yacón del banco de germoplasma del INIA fueron seleccionadas por sus atributos agronómicos, nutricionales y nutracéuticos (Tabla 1 y 2). La siembra se realizó en Junio del 2011 en la comunidad de Rosapata (10¹1'09"S, 7609'13"W) ubicada a $2792 \mathrm{~m}$ de altitud en Ambo, Huánuco. Cada una de las seis accesiones seleccionadas se sembró en cuatro parcelas, con 60 plantas cada una. Los híbridos fueron obtenidos previamente a partir del cruzamiento de los seis parentales de yacón usando un diseño de cruzas dialélicas (Manrique et al. 2014).

De las 162 plantas obtenidas a partir de la regeneración de las semillas, se seleccionaron 68 , con sus respectivas repeticiones (en total 834 individuos, Tabla 3 y 4), debido a que estas fueron las únicas de las cuales se conocía el origen de sus parentales, para realizar la caracterización morfológica y determinar si realmente son el resultado de un proceso de hibridación.

Tabla 1. Datos pasaporte y algunos atributos de interés de los parentales del yacón, Smallanthus sonchifolius, utilizadas en el estudio de caracterización morfológica de seis variedades parentales y trece cruzas.

\begin{tabular}{|c|c|c|c|c|}
\hline Código & Código colector & Altitud (msnm) & Localidad & Descripción \\
\hline Testigo & Testigo & - & Bagua & Yacón blanco-Variedad control \\
\hline V4 & UNALM 2 & - & Huánuco & $\begin{array}{l}\text { Alta productividad de raíces, alto Brix, materia seca y } \\
\text { FOS. }\end{array}$ \\
\hline V11 & ZSY044 & 1200 & San Gaban, Carabaya, Puno & $\begin{array}{l}\text { Tiene antocianinas y alta actividad antioxidante.Yacón } \\
\text { amarillo }\end{array}$ \\
\hline V18 & PER007663 & 1820 & Sivia, Huanta, Ayacucho & Resistente al pardeamiento. \\
\hline V24 & AMM5163 & 3400 & Shilla, Carhuaz, Ancash & $\begin{array}{l}\text { Alto contenido de Brix, materia seca, FOS y compuestos } \\
\text { fenólicos. }\end{array}$ \\
\hline V28 & UNALM4 & - & Huánuco & Alta productividad de raíces y alto FOS. \\
\hline
\end{tabular}


Tabla 2. Caracterización morfológica de las 6 accesiones parentales de yacón

\begin{tabular}{|c|c|c|c|}
\hline D & TESTIGO (Smallanthus sonchifolius) & V4 (Smallanthus sonchifolius) & V11 (Smallanthus sonchifolius) \\
\hline D1 & Tallo verde amarillento & Tallo púrpura grisáceo oscuro & Tallo verde amarillento \\
\hline D2 & Nudos y entrenudos púrpuras & Sin color en nudos o entrenudos & Nudos y entrenudos púrpuras \\
\hline D3 & Ramificación a través de todo el tallo & Ramificación a través de todo el tallo & Ramificación a través de todo el tallo \\
\hline D4 & Follaje verde amarillento & Follaje verde & Follaje verde amarillento \\
\hline D5 & Sin pigmentación en la nervadura de la hoja & Sin pigmentación en la nervadura de la hoja & Sin pigmentación en la nervadura de la hoja \\
\hline D6 & Sin pigmentación en la hoja apical & Sin pigmentación en la hoja apical & Sin pigmentación en la hoja apical \\
\hline D7 & Hoja de forma Triangular-Hastada & Hoja de forma Triangular-Sub hastada & Hoja de forma Triangular-Hastada \\
\hline D8 & Borde de la hoja: Dentado & Borde de la hoja: Dentado & Borde de la hoja: Dentado \\
\hline D9 & Con floración moderada & Con floración abundante & Con floración abundante \\
\hline D10 & Flor ligulada amarillo naranja & Flor ligulada amarillo naranja & Flor ligulada amarillo naranja \\
\hline D11 & Flor ligulada de forma oblonga & Flor ligulada de forma oblonga & Flor ligulada de forma oblonga \\
\hline D12 & Flor ligulada bidentada & Flor ligulada tridentada & Flor ligulada bidentada \\
\hline D13 & Si produce semilla & Si produce semilla & Si produce semilla \\
\hline D14 & Superficie de la raíz púrpura grisáceo claro & Superficie de la raíz púrpura grisáceo oscuro & Superficie de la raíz amarillo claro \\
\hline D15 & Pulpa de la raíz blanca & Pulpa de la raíz amarillo naranja & $\begin{array}{l}\text { Pulpa de la raíz blanco amarillento con mo- } \\
\text { teaduras irregulares púrpura rojizo }\end{array}$ \\
\hline D16 & No presenta hendiduras en la raíz (lisa) & No presenta hendiduras en la raíz (lisa) & Presenta hendiduras en la raíz \\
\hline D17 & Propágulo blanco con rojo purpúreo & Propágulo purpúra grisáceo oscuro & Propágulo blanco con rojo purpúreo \\
\hline
\end{tabular}

\begin{tabular}{|c|c|c|c|}
\hline D & V18 (Smallanthus sonchifolius) & V24 (Smallanthus sonchifolius) & V28 (Smallanthus sonchifolius) \\
\hline D1 & Tallo púrpura grisáceo oscuro & Tallo púrpura grisáceo oscuro & Tallo verde amarillento \\
\hline D2 & Sin color en nudos o entrenudos & Sin color en nudos o entrenudos & Nudos y entrenudos púrpuras \\
\hline D3 & Ramificación a través de todo el tallo & Ramificación a través de todo el tallo & Ramificación a través de todo el tallo \\
\hline D4 & Follaje verde amarillento & Follaje verde & Follaje verde amarillento \\
\hline D5 & Sin pigmentación en la nervadura de la hoja & Con pigmentación en la nervadura de la hoja & Sin pigmentación en la nervadura de la hoja \\
\hline D6 & Sin pigmentación en la hoja apical & Sin pigmentación en la hoja apical & Sin pigmentación en la hoja apical \\
\hline D7 & Hoja de forma Triangular-Hastada & Hoja de forma Triangular-Hastada & Hoja de forma Triangular-Hastada \\
\hline D8 & Borde de la hoja: Dentado & Borde de la hoja: Dentado & Borde de la hoja: Dentado \\
\hline D9 & Con floración abundante & Con floración escasa & Con floración abundante \\
\hline D10 & Flor ligulada amarillo naranja & Sin flor & Flor ligulada amarillo naranja \\
\hline D11 & Flor ligulada de forma oblonga & Sin flor & Flor ligulada de forma elíptica \\
\hline D12 & Flor ligulada tridentada & Sin flor & Flor ligulada bidentada \\
\hline D13 & Si produce semilla & Si produce semilla & Si produce semilla \\
\hline D14 & Superficie de la raíz púrpura grisáceo claro & Superficie de la raíz púrpura grisáceo oscuro & Superficie de la raíz amarillo claro \\
\hline D15 & Pulpa de la raíz naranja claro & Pulpa de la raíz amarillo naranja & Pulpa de la raíz amarillo naranja \\
\hline D16 & Presenta hendiduras en la raíz & Presenta hendiduras en la raíz & No presenta hendiduras en la raíz (lisa) \\
\hline D17 & Propágulo púrpura grisáceo oscuro & Propágulo rojo purpúreo & Propágulo púrpura grisáceo oscuro \\
\hline
\end{tabular}


Tabla 3. Cruzamientos realizados, tipos de cruzas y número de híbridos obtenidos en campo.

\begin{tabular}{ccc}
\hline Cruzamiento & Tipo de cruza & $\begin{array}{c}\text { Total de híbridos viables } \\
\text { evaluados por cada tipo de } \\
\text { cruzamiento }\end{array}$ \\
\hline V4 x V11 & CD & 2 \\
V4 x V28 & CD & 3 \\
V11 x V4 & CR & 4 \\
V11 x V18 & CD & 3 \\
V11 x V24 & CD & 16 \\
V11 x V28 & CD & 2 \\
V11 x Testigo & CR & 4 \\
V24 x V4 & CR & 4 \\
V24 x V11 & CR & 9 \\
V24 x V18 & CR & 7 \\
V24 x V28 & CD & 4 \\
V24 x Testigo & CR & 6 \\
Testigo x V11 & CD & 4 \\
\hline TOTAL & & 68 \\
\hline
\end{tabular}

Caracterización morfológica.- Se empleó la lista de 17 descriptores morfológicos para yacón de Arbizu et al. (2001). Los descriptores de color fueron evaluados con una cartilla de colores (RHS 2007).

La caracterización de los parentales se realizó en abril del 2013, y se evaluaron 10 plantas de cada parental y se emplearon todos los descriptores. La caracterización de los híbridos se realizó en marzo del 2015, y se evaluaron cinco plantas por cada híbrido. Únicamente se evaluaron tres descriptores morfológicos en los híbridos: color de la superficie de la raíz (D14), color de la pulpa de la raíz (D15) y tendencia a formar hendiduras en la raíz (D16).

Después de concluida la caracterización morfológica, los estados de los tres descriptores evaluados para cada híbrido fueron comparados con los estados de cada uno de sus parentales; para esto se tomaron en cuenta variantes respecto a los colores, considerando el púrpura grisáceo oscuro como púrpura grisáceo claro, el amarillo naranja como amarillo claro y el blanco amarillento como blanco, ya que al realizar la caracterización se observó similitud entre estos, lo que permitió una mejor discriminación entre los híbridos.

Se determinaron cuatro categorías para clasificar a los 68 individuos obtenidos: si el híbrido expresó caracteres de ambos progenitores, fue considerado un "híbrido positivo"; si fue híbrido pero solo expresó caracteres del progenitor femenino o no existió hibridación, entonces fue considerado un "no determinado"; si ambos progenitores presentan caracteres compartidos y debido a esto no se puede determinar si heredó características de ambos o solo del progenitor femenino o no existió hibridación, entonces fue considerado un "no específico"; si el individuo presentó características diferentes a ambos progenitores, entonces fue considerado un "alterado".

La caracterización morfológica comprendió la elaboración del catálogo fotográfico de los parentales (Anexo 1) y de los híbridos (Anexo 2).
Tabla 4. Número de plantas obtenidas por cada cruzamiento.

\begin{tabular}{|c|c|c|}
\hline Cruzamiento & Código & $\mathrm{N}^{o}$ de plantas \\
\hline \multirow{2}{*}{ V4 x V11 } & 1.1 & 3 \\
\hline & 1.2 & 2 \\
\hline \multirow{3}{*}{$\mathrm{V} 4 \times \mathrm{V} 28$} & 2.1 & 13 \\
\hline & 2.2 & 21 \\
\hline & 2.3 & 2 \\
\hline \multirow{4}{*}{$\mathrm{V} 11 \times \mathrm{V} 4$} & 3.1 & 17 \\
\hline & 3.2 & 7 \\
\hline & 3.3 & 11 \\
\hline & 3.4 & 11 \\
\hline \multirow{3}{*}{ V11 x V18 } & 4.1 & 13 \\
\hline & 4.2 & 3 \\
\hline & 4.3 & 9 \\
\hline \multirow{16}{*}{$\mathrm{V} 11 \times \mathrm{V} 24$} & 5.1 & 4 \\
\hline & 5.2 & 4 \\
\hline & 5.3 & 2 \\
\hline & 5.4 & 4 \\
\hline & 5.5 & 6 \\
\hline & 5.6 & 14 \\
\hline & 5.7 & 13 \\
\hline & 5.8 & 22 \\
\hline & 5.9 & 18 \\
\hline & 5.10 & 8 \\
\hline & 5.11 & 24 \\
\hline & 5.12 & 25 \\
\hline & 5.13 & 12 \\
\hline & 5.14 & 11 \\
\hline & 5.15 & 23 \\
\hline & 5.16 & 4 \\
\hline \multirow{2}{*}{$\mathrm{V} 11 \times \mathrm{V} 28$} & 6.1 & 2 \\
\hline & 6.2 & 8 \\
\hline \multirow{4}{*}{ V11 $\times$ Testigo } & 7.1 & 27 \\
\hline & 7.2 & 3 \\
\hline & 7.3 & 2 \\
\hline & 7.4 & 15 \\
\hline \multirow{4}{*}{$\mathrm{V} 24 \times \mathrm{V} 4$} & 8.1 & 35 \\
\hline & 8.2 & 25 \\
\hline & 8.3 & 25 \\
\hline & 8.4 & 10 \\
\hline \multirow{9}{*}{ V24 x V11 } & 9.1 & 7 \\
\hline & 9.2 & 7 \\
\hline & 9.3 & 5 \\
\hline & 9.4 & 23 \\
\hline & 9.5 & 13 \\
\hline & 9.6 & 15 \\
\hline & 9.7 & 18 \\
\hline & 9.8 & 19 \\
\hline & 9.9 & 11 \\
\hline \multirow{7}{*}{ V24 x V18 } & 10.1 & 8 \\
\hline & 10.2 & 15 \\
\hline & 10.3 & 20 \\
\hline & 10.4 & 8 \\
\hline & 10.5 & 18 \\
\hline & 10.6 & 8 \\
\hline & 10.7 & 34 \\
\hline \multirow{4}{*}{$\mathrm{V} 24 \times \mathrm{V} 28$} & 11.1 & 27 \\
\hline & 11.2 & 8 \\
\hline & 11.3 & 5 \\
\hline & 11.4 & 19 \\
\hline \multirow{6}{*}{ V24 x Testigo } & 12.1 & 6 \\
\hline & 12.2 & 13 \\
\hline & 12.3 & 11 \\
\hline & 12.4 & 4 \\
\hline & 12.5 & 15 \\
\hline & 12.6 & 12 \\
\hline \multirow{5}{*}{ Testigo x V11 } & 13.1 & 6 \\
\hline & 13.2 & 5 \\
\hline & 13.3 & 8 \\
\hline & 13.4 & 8 \\
\hline & & 834 \\
\hline
\end{tabular}


Análisis morfológico.- Se realizó el Análisis de Conglomerados, empleando el índice de Jaccard para determinar el estado de hibridación, de acuerdo a los valores de las variantes de cada descriptor. Para el análisis se utilizó el programa InfoStat (http:// www.infostat.com.ar/).

\section{Resultados}

Los resultados de la caracterización morfológica para los 17 descriptores de los seis parentales son mostrados en la Tabla 2 y Anexo 1. De los 68 individuos obtenidos a partir de las cruzas, de acuerdo a la caracterización morfológica y al análisis de conglomerados, se obtuvieron 44 "híbridos positivos"; 8 "no determinados", ya que solo heredaron los caracteres del progenitor femenino, no logrando identificarse si existió hibridación o no; 9 "no específicos", ya que al presentar los progenitores caracteres compartidos, pudo existir hibridación heredando las características de ambos o solo del progenitor femenino o no existir; 7 "alterados", ya que el progenitor masculino no es el correspondiente a la cruza; 2 replicaciones de 3 "híbridos positivos" y 1 replicación de 1 de los 9 "no específicos" también presentaron un progenitor masculino diferente al de su cruza considerándoseles "alterados" (Tabla 5 y Anexo 2).

Se observó precocidad en el parental V24, el inicio y la finalización de la floración ocurrió mucho antes en comparación los otros parentales. Además, se observó la pérdida de moteaduras irregulares púrpura rojizo (indicador de antocianinas) en las 44 plantas con parentales que presentaban este carácter.

\section{Discusión}

La caracterización morfológica de una población de híbridos de yacón y sus respectivos parentales, permitió diferenciarlos y determinar su naturaleza híbrida.

Para la caracterización morfológica de los parentales e híbridos se emplearon 10 y 5 individuos de cada uno, respectivamente. En el caso de los híbridos se empleó este número de muestra por la condición en que se encontraron las raíces al momento de la cosecha; sin embargo, de acuerdo a la Unión Internacional para la Protección de las Obtenciones Vegetales 2011 (UPOV 2011), para medir la variabilidad de una población, en este caso híbridos y parentales, es necesario utilizar descriptores discriminatorios, como los de Arbizu et al. (2001) y establecer el experimento con un mínimo de cinco plantas por accesión en lotes homogéneos en dos replicaciones, de este modo se obtendrá mejor y mayor información (Hernández 2013).

Por lo general, los híbridos presentan características físicas intermedias, por lo cual se determinó como status de hibridación "positivo" a aquellas plantas que cumplen este patrón (Dorado 2008). Sin embargo, en algunos casos, se observa la morfología intermedia entre los dos parentales, donde el híbrido se parece a uno de los padres más que el otro o se ve diferente a cualquiera de los padres (Ponton et al. 2004, Ito 2009). La expresión del carácter parental o caracteres intermedios en los híbridos depende del control genético y la influencia del ambiente (Rieseberg 1995).

Las especies de tubérculos como la papa, presentan barreras internas pre-cigóticas y post-cigóticas, estas pueden ser unilaterales o bilaterales, según ocurran en uno o en ambos sentidos de un mismo cruzamiento; cuando están ausentes o son incompletas, se puede originar progenie híbrida (Camadro 2011). En los híbridos de yacón obtenidos, una o ambas barreras se encontraron inactivas generando un status de hibridación "positivo"; mientras que en los status de "no determinado" y "no específico", no podría afirmarse de forma concluyente si dichas barreras se manifestaron o no, ya que por ejemplo, en el primer caso, los individuos pueden ser progenie híbrida que solo heredó características de su progenitor femenino o no ser híbridos, siendo en este panorama una copia de la variedad que se empleó como progenitor femenino; en el segundo caso, al ser los parentales similares, pueden ser híbridos que heredaron características que el parental masculino compartía con el femenino o ser solo copias de la variedad que se empleó como progenitor femenino. En cambio, en el status de hibridación "alterada", se habría generado una progenie híbrida, pero teniendo como parental masculino una planta diferente a la establecida en el plan de cruzamiento, siendo una o ambas barreras inactivadas antes de la ejecución del plan de cruzamiento. Para determinar, si la progenie en los status de "no determinada" y "no específica" es híbrida, se recomienda realizar una caracterización molecular empleando marcadores AFLPs.

Es evidente que en los híbridos el carácter de color de superficie púrpura grisáceo oscuro es dominantes (ver Tabla 4), ya que cuando está presente en el progenitor masculino mas no en el femenino, el híbrido expresa este carácter, de igual forma cuando está presente en el progenitor femenino mas no en el masculino.

El carácter de moteaduras irregulares púrpura rojizo es recesivo, ya que todos los híbridos no expresaron el carácter. Sin embargo, de acuerdo a los resultados del trabajo de Sidorov et al. (1987), la síntesis de antocianina en híbridos somáticos de papa se expresa, en lo que ellos denominan los verdaderos híbridos y por lo tanto es un carácter dominante. Además, Mano et al., 2007 reportan en camote, que la expresión de antocianinas en varios tejidos de la planta es de tipo dominante. Observándose que la expresión de este carácter varía de acuerdo a la especie.

El carácter de presencia de hendiduras en la raíz es recesivo, y se observó que depende del sustrato de las plantas.

La floración es intensa y precoz en la mayoría de los clones de yacón del norte de Bolivia, alrededores de Cusco, sur y norte del Perú, y Cajamarca, tal como también se evidencia en el parental V24 que resultó ser precoz, y es originario de la región de Ancash (Singh 2012). Además, V24 representa una ventaja para este cultivo, ya que, permitiría erradicar estos genotipos del campo antes que alcance la antesis y así evitar sus posibles recombinaciones con las demás variedades (Ortiz et al. 2008). Además, el ciclo precoz permite que, con fechas de siembra tempranas, transcurran su período crítico (alrededor de floración) durante épocas de menor demanda atmosférica (ambiente menos "desecante") que plantas de ciclo más largo. Teniendo menor probabilidad de estar expuestos a déficit hídrico durante el período crítico. Además, las plantas de ciclo corto, requieren de buenos ambientes para expresar todo su potencial; sin embargo, se ha observado que las plantas V24 tuvieron un óptimo crecimiento en las condiciones de Ambo, Huánuco, siendo este un buen ambiente no solo para el desarrollo de los parentales sino también para el desarrollo de los híbridos.

\section{Agradecimientos}

Al Instituto Nacional de Innovación Agraria (INIA) por proveer las accesiones de yacón que se usaron como parentales en las cruzas dialélicas. 
Tabla 5. Caracterización morfológica de las 72 accesiones híbridas de acuerdo a los descriptores morfológicos de Arbizu et al, 2001 y el status de hibridación. $A=$ Ausente; $P$ = Presente; $P G O=$ Púrpura grisáceo oscuro; $P G C$ = Púrpura grisáceo claro; $A N$ = Amarillo naranja; NC = Naranja claro; $\mathrm{B}=$ Blanco; $\mathrm{BA}=$ Blanco amarillento; BA-MPR = Blanco amarillento con moteaduras irregulares púrpura rojizo; $\mathrm{AV}=$ Azúl violeta.

\begin{tabular}{|c|c|c|c|c|c|c|c|c|c|c|c|c|}
\hline 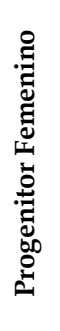 & 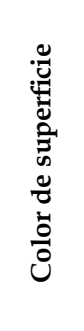 & 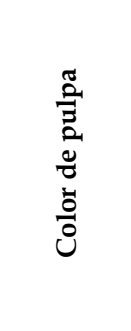 & 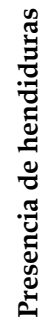 & 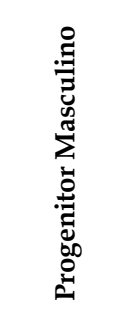 & 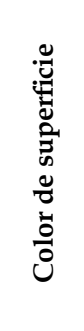 & $\begin{array}{l}\frac{a}{2} \\
\frac{a}{2} \\
\frac{0}{0} \\
\frac{0}{0} \\
0\end{array}$ & 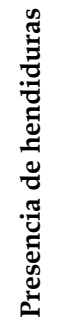 & $\begin{array}{l}0 \\
0 \\
0 \\
0 \\
0 \\
0 \\
0 \\
0 \\
0 \\
0 \\
: 0 \\
0 \\
0\end{array}$ & 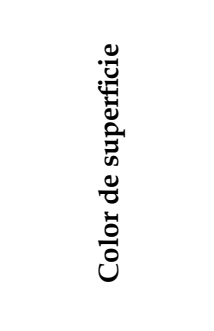 & $\begin{array}{l}\frac{a}{2} \\
\frac{0}{2} \\
\frac{0}{0} \\
\frac{0}{0}\end{array}$ & 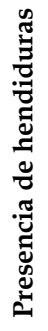 & 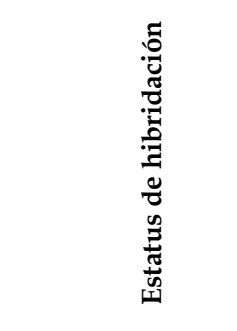 \\
\hline \multirow{2}{*}{ V4 } & \multirow{2}{*}{ PGO } & \multirow{2}{*}{ AN } & \multirow{2}{*}{ A } & \multirow{2}{*}{ V11 } & \multirow{2}{*}{$\mathrm{AC}$} & \multirow{2}{*}{$\begin{array}{l}\text { BA- } \\
\text { MPR }\end{array}$} & \multirow{2}{*}{$\mathrm{P}$} & 1.1 & PGO & $\mathrm{NC}$ & $\mathrm{A}$ & No determinado \\
\hline & & & & & & & & 1.2 & PGO & $\mathrm{NC}$ & $\mathrm{A}$ & No determinado \\
\hline \multirow{3}{*}{ V4 } & \multirow{3}{*}{ PGO } & \multirow{3}{*}{ AN } & \multirow{3}{*}{$\mathrm{A}$} & \multirow{3}{*}{ V28 } & \multirow{3}{*}{$\mathrm{AC}$} & \multirow{3}{*}{ AN } & \multirow{3}{*}{ A } & 2.1 & PGO & $\mathrm{NC}$ & $\mathrm{A}$ & No específico \\
\hline & & & & & & & & 2.2 & PGO & $\mathrm{BA}$ & $\mathrm{A}$ & Alterada \\
\hline & & & & & & & & 2.3 & PGO & $\mathrm{AN} / \mathrm{NC}$ & $\mathrm{A}$ & No específico \\
\hline \multirow{4}{*}{ V11 } & \multirow{4}{*}{$\mathrm{AC}$} & \multirow{4}{*}{ BA-MPR } & \multirow{4}{*}{$\mathrm{P}$} & \multirow{4}{*}{ V4 } & \multirow{4}{*}{ PGO } & \multirow{4}{*}{ AN } & & 3.1 & PGO & $\mathrm{AC}$ & $\mathrm{A}$ & Positivo \\
\hline & & & & & & & $A$ & 3.2 & $\mathrm{AC}$ & BA & A & Positivo \\
\hline & & & & & & & $\mathrm{A}$ & 3.3 & PGO & $\mathrm{AC}$ & $\mathrm{P}$ & Positivo \\
\hline & & & & & & & & 3.4 & $\mathrm{AC}$ & BA & $\mathrm{A}$ & Positivo \\
\hline & & & & & & & & 4.1 & PGO & $\mathrm{NC}$ & $\mathrm{A}$ & Positivo \\
\hline V11 & $\mathrm{AC}$ & BA-MPR & $\mathrm{P}$ & V18 & PGC & NC & $\mathrm{P}$ & 4.2 & AC/PGC & $\mathrm{BA} / \mathrm{AC}$ & $\mathrm{A}$ & Positivo \\
\hline & & & & & & & & 4.3 & AC/PGC/PGO & $\mathrm{BA} / \mathrm{NC}$ & A & Positivo \\
\hline & & & & & & & & 5.1 & PGC/PGO & $\mathrm{B} / \mathrm{NC}$ & A & Positivo \\
\hline & & & & & & & & 5.2 & PGC/PGO/AV & $\begin{array}{c}\mathrm{BA} / \mathrm{AN} / \\
\mathrm{NC}\end{array}$ & A & Positivo \\
\hline & & & & & & & & 5.3 & PGC & BA & $\mathrm{A}$ & Positivo \\
\hline & & & & & & & & 5.4 & $\mathrm{AC}$ & B & $\mathrm{A}$ & No determinado \\
\hline & & & & & & & & 5.5 & PGO & $\mathrm{NC}$ & A & Positivo \\
\hline & & & & & & & & 5.6 & PGO & AN & $\mathrm{A}$ & Positivo \\
\hline & & & & & & & & 5.7 & PGO & AN & $\mathrm{P}$ & Positivo \\
\hline V11 & $\Delta C$ & PA MPD & $\mathrm{P}$ & $V 24$ & $\mathrm{PCO}$ & N & $\mathrm{P}$ & 5.8 & PGC & $\mathrm{AN} / \mathrm{NC}$ & $\mathrm{A}$ & Positivo \\
\hline$\sqrt{11}$ & AC & DA-IVII K & 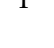 & $\sqrt{ }<4$ & 100 & AIV & 1 & 5.9 & PGO & $\mathrm{NC}$ & $\mathrm{A}$ & Positivo \\
\hline & & & & & & & & 5.10 & PGO & AN & $\mathrm{A}$ & Positivo \\
\hline & & & & & & & & 5.11 & PGC/PGO & $\mathrm{AN} / \mathrm{NC}$ & A & Positivo \\
\hline & & & & & & & & 5.12 & PGO & AN/NC & $\mathrm{A}$ & Positivo \\
\hline & & & & & & & & 5.13 & PGO & $\mathrm{AC}$ & $\mathrm{P}$ & Positivo \\
\hline & & & & & & & & 5.14 & PGO & $\mathrm{AN} / \mathrm{NC}$ & $\mathrm{A}$ & Positivo \\
\hline & & & & & & & & 5.15 & PGO & $\mathrm{B} / \mathrm{AN} / \mathrm{NC}$ & $\mathrm{A}$ & Positivo \\
\hline & & & & & & & & 5.16 & PGC & $\mathrm{NC}$ & $\mathrm{A}$ & Positivo \\
\hline$V 11$ & $\Delta C$ & PA MPP & $\mathrm{P}$ & Vרe & $\Delta$ & $\mathrm{NI}$ & $A$ & 6.1 & $\mathrm{AC}$ & $\mathrm{AC}$ & A & Positivo \\
\hline$\sqrt{11}$ & $\mathrm{AC}$ & DA-IVIIK & $P$ & $v \angle 8$ & $\mathrm{AC}$ & AIN & A & 6.2 & $\mathrm{AC}$ & BA & $\mathrm{A}$ & Positivo \\
\hline & & & & & & & & 7.1 & PGC & $\mathrm{BA} / \mathrm{NC}$ & $\mathrm{A}$ & Positivo/Alterada \\
\hline $\mathrm{V} 11$ & $\Delta C$ & $\mathrm{~B} \triangle \mathrm{MPP}$ & $\mathrm{P}$ & Toction & $\mathrm{PCC}$ & $R_{2}$ & $\Delta$ & 7.2 & PGC & $\mathrm{NC}$ & $\mathrm{A}$ & Alterada \\
\hline$\sqrt{ } 11$ & $\mathrm{AC}$ & ВА-МПРК & $P$ & lestigo & PGC & B & A & 7.3 & PGC & $\mathrm{NC}$ & $\mathrm{A}$ & Alterada \\
\hline & & & & & & & & 7.4 & PGO & $\mathrm{NC}$ & A & Alterada \\
\hline & & & & & & & & 8.1 & PGO & AN & A & Positivo \\
\hline V24 & PCO & $A \mathrm{NI}$ & $\mathrm{P}$ & $V A$ & $\mathrm{PCO}$ & NT & $A$ & 8.2 & PGO & BA & $\mathrm{A}$ & Alterada \\
\hline$\sqrt{ } 24$ & PGO & AN & $P$ & $\sqrt{ } 4$ & PGO & AN & A & 8.3 & PGO & BA & $\mathrm{A}$ & Alterada \\
\hline & & & & & & & & 8.4 & PGO & $\mathrm{NC}$ & $\mathrm{A}$ & Positivo \\
\hline
\end{tabular}


Tabla 5. Continuación ...

\begin{tabular}{|c|c|c|c|c|c|c|c|c|c|c|c|c|}
\hline 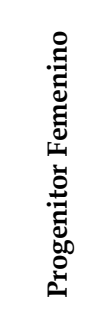 & 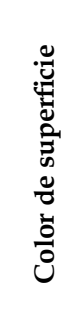 & 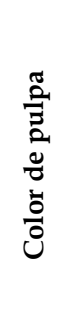 & 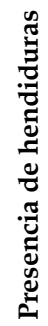 & 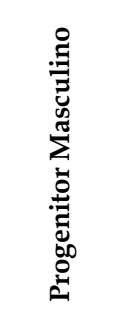 & 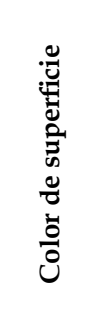 & 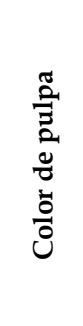 & 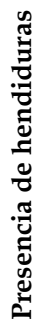 & 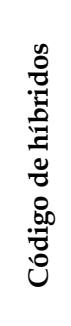 & 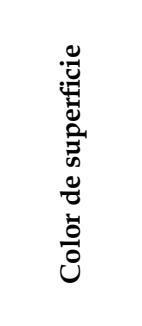 & 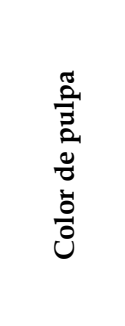 & 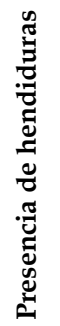 & 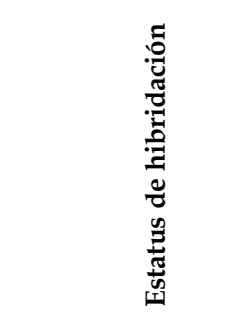 \\
\hline \multirow{9}{*}{ V24 } & \multirow{9}{*}{ PGO } & \multirow{9}{*}{ AN } & \multirow{9}{*}{$\mathrm{P}$} & \multirow{9}{*}{ V11 } & \multirow{9}{*}{$\mathrm{AC}$} & \multirow{9}{*}{$\begin{array}{l}\text { BA- } \\
\text { MPR }\end{array}$} & \multirow{9}{*}{$\mathrm{P}$} & 9.1 & PGC & $\mathrm{NC}$ & $\mathrm{A}$ & No determinado \\
\hline & & & & & & & & 9.2 & PGC & NC & $\mathrm{A}$ & No determinado \\
\hline & & & & & & & & 9.3 & PGO & $\mathrm{BA} / \mathrm{AN}$ & $\mathrm{A}$ & Positivo \\
\hline & & & & & & & & 9.4 & PGO & AN & A & No determinado \\
\hline & & & & & & & & 9.5 & PGO & $\mathrm{BA} / \mathrm{AN}$ & A & Positivo \\
\hline & & & & & & & & 9.6 & $\mathrm{AC}$ & AN & $\mathrm{P}$ & Positivo \\
\hline & & & & & & & & 9.7 & PGO & $\mathrm{BA} / \mathrm{AN}$ & $\mathrm{A}$ & Positivo \\
\hline & & & & & & & & 9.8 & PGO & $\mathrm{NC}$ & A & No determinado \\
\hline & & & & & & & & 9.9 & PGO & $\mathrm{AN} / \mathrm{NC}$ & $\mathrm{A}$ & No determinado \\
\hline \multirow{7}{*}{ V24 } & \multirow{7}{*}{ PGO } & \multirow{7}{*}{ AN } & \multirow{7}{*}{$\mathrm{P}$} & \multirow{7}{*}{ V18 } & \multirow{7}{*}{ PGC } & \multirow{7}{*}{$\mathrm{NC}$} & \multirow{7}{*}{$\mathrm{P}$} & 10.1 & AC/PGO & $\mathrm{AN} / \mathrm{NC}$ & A & $\begin{array}{l}\text { Alterado/No } \\
\text { específico }\end{array}$ \\
\hline & & & & & & & & 10.2 & PGO & $\mathrm{NC}$ & $\mathrm{A}$ & No específico \\
\hline & & & & & & & & 10.3 & PGO & AN & A & No específico \\
\hline & & & & & & & & 10.4 & PGO & $\mathrm{AC}$ & $\mathrm{A}$ & No específico \\
\hline & & & & & & & & 10.5 & PGO & $\mathrm{NC}$ & $\mathrm{A}$ & No específico \\
\hline & & & & & & & & 10.6 & PGO & $\mathrm{AN} / \mathrm{NC}$ & $\mathrm{A}$ & No específico \\
\hline & & & & & & & & 10.7 & PGO & $\mathrm{NC}$ & $\mathrm{A}$ & No específico \\
\hline \multirow{4}{*}{ V24 } & \multirow{4}{*}{ PGO } & \multirow{4}{*}{ AN } & \multirow{4}{*}{$\mathrm{P}$} & \multirow{4}{*}{ V28 } & \multirow{4}{*}{$\mathrm{AC}$} & \multirow{4}{*}{ AN } & \multirow{4}{*}{ A } & 11.1 & AC/PGO & $\mathrm{BA} / \mathrm{AN}$ & $\mathrm{A}$ & Alterada/Positivo \\
\hline & & & & & & & & 11.2 & PGO & AN & A & Positivo \\
\hline & & & & & & & & 11.3 & PGC & $\mathrm{NC}$ & $\mathrm{A}$ & Positivo \\
\hline & & & & & & & & 11.4 & PGO & $\mathrm{AN} / \mathrm{NC}$ & $\mathrm{A}$ & Positivo \\
\hline \multirow{6}{*}{ V24 } & \multirow{6}{*}{ PGO } & \multirow{6}{*}{ AN } & & & & & & 12.1 & PGO & AN & A & Positivo \\
\hline & & & & & & & & 12.2 & PGO & $\mathrm{AN} / \mathrm{NC}$ & $\mathrm{A}$ & Positivo \\
\hline & & & $\mathrm{P}$ & TTotio & $\mathrm{PCC}$ & $\mathrm{P}$ & $\Lambda$ & 12.3 & PGC & BA & $\mathrm{A}$ & Positivo \\
\hline & & & 1 & resugo & $1 \in$ & D & $\mathrm{A}$ & 12.4 & PGO & $\mathrm{NC}$ & $\mathrm{A}$ & Positivo \\
\hline & & & & & & & & 12.5 & PGO & $\mathrm{NC}$ & $\mathrm{A}$ & Positivo \\
\hline & & & & & & & & 12.6 & PGO & $\mathrm{NC}$ & $\mathrm{A}$ & Positivo \\
\hline & & & & & & & & 13.1 & PGC & $\mathrm{AC}$ & $\mathrm{A}$ & Positivo \\
\hline Tectior & PCC & $\mathrm{B}_{\mathrm{B}}$ & 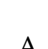 & $\mathrm{V} 11$ & $\Delta C$ & BA- & $\mathrm{P}$ & 13.2 & PGC & BA & A & Positivo \\
\hline iestigo & 10 & D & $\mathrm{A}$ & $\sqrt{11}$ & AC & MPR & 1 & 13.3 & AC/PGO & $\mathrm{AC} / \mathrm{AN}$ & $\mathrm{A}$ & Positivo/Alterada \\
\hline & & & & & & & & 13.4 & AC & NC & A & Alterada \\
\hline
\end{tabular}

\section{Literatura citada}

Arbizu, C., R. Blas, M. Holle, F. Vivanco \& M. Ghislain. 1997. Advances in the morphological characterization of oca, ulluco, mashua, and arracacha Collections. The International Potato Center, Program Report. Lima, Perú. p.110-117.

Camadro E. 2011. Hibridación y flujo génico en especies silvestres de papa de la Argentina. Journal of Basic \& Applied Genetics. 22(1): 1-5.

Campos D., I. Betalleluz-Pallardel, R. Chirinos, A. Aguilar-Galvez, G. Noratto \& R. Pedreschi. 2012. Prebiotic effects of yacón (Smallanthus sonchifolius Poepp. \& Endl), a source of fructooligosaccharides and phenolic compounds with antioxidant activity. Journal of Food Chemistry. 135: 1592-1599.
Coronado A. 2013. Elaboración de la harina de yacón (Smallanthus sonchifolius) y su influencia en el crecimiento de dos bacterias probióticas. Tesis para el Título de Químico Farmacéutico. Facultad de Farmacia y Bioquímica Universidad Nacional Mayor de San Marcos. Lima, Perú: Universidad Nacional Mayor de San Marcos. http://cybertesis.unmsm. edu.pe/handle/cybertesis/3201

Dorado O. 2008. Hibridación: ¿promiscuidad biológica?, en Academia de Ciencia de Morelos, A.C, pp. 22-23 http://acmor.org. $\mathrm{mx} /$ descargas/08_oct_20_hibridacion.pdf

Franco T.L. \& R. Hidalgo. 2003. Análisis Estadístico de Datos de Caracterización Morfológica de Recursos Fitogenéticos. Boletín técnico del Instituto Internacional de Recursos Fitogenéticos (IPGRI). 8: 89p. 
Grau A. \& J. Rea. 1997. Yacón, Smallanthus sonchifolius (Poepp. \& Endl) H. Robinson. In: Hermann M. \& J. Heller (eds): Andean roots and tuber: Ahipa, arracacha, maca, yacón. IPGRI, Roma Italia. Pp. 199-242.

Hernandez A.E. 2013. Caracterización Morfológica de Recursos Fitogenéticos. Revista Bio Ciencias. 2(3): 113-118.

Ito M. 2009. Variation in leaf morphology of Quercus crispula and Quercus dentata assemblages among contact zones: a method for detection of probable hybridization. Journal of Forest Research. 14:240-244.

Laguna C.A., M.E. Guadarrama-Guadarrama, Y.R.Arenas-Julio \& M.R. Delgado. 2006. Aplicación de la guía de descripción varietal de dalia (Dahlia spp) en la caracterización de clones seleccionados. Ciencias Agrícolas Informa. 4: 24-29.

Mano H., F. Ogasawara, K. Sato, H. Higo \& Y. Minobe. 2007. Isolation of a Regulatory Gene of Anthocyanin Biosynthesis in Tuberous Roots of Purple-Fleshed Sweet Potato. Plant Physiology. 143:1252-1268.

Manrique I., R. Gonzales, A. Valladolid, R. Blas \& L. Lizárraga. 2014. Producción de semillas en yacón (Smallanthus sonchifolius (Poepp. \& Endl.)) mediante técnicas de polinización controladas. Journal of Ecología Aplicada. 13(2): 135-145.

Mansilla R., C. López, M. Flores \& R. Espejo. 2010. Estudio de la Biología Reproductiva en cinco accesiones de Smallanthus sonchifolius (Poepp. \& Endl.) Robinson. Journal of Ecología Aplicada. 9(2): 167-175.

Milella L., J. Salava, G. Martelli, et al. 2005. Genetic diversity between yacón landraces from different countries based on random amplified polymorphic DNAs. Czech Journal of Genetics and Plant Breeding 41:73-78

Ortiz A., R. Miranda, R. Figueroa \& C. Ramis. 2008. Caracterización Morfológica de una población F2 obtenida del cruce natural entre un cultivar arroz y un arroz rojo (Parte I). Agronomía Tropical. 58(3): 299-307.

Ponton S., J.L. Dupouey \& E. Dreyer. 2004. Leaf morphology as species indicator in seedlings of Quercus robur L. and Q. petraea (Matt.) Liebl: modulation by irradiance and growth flush. Annals of Forest Science. 61: 73-80.

Rieseberg L.H. 1995. The role of hybridization in evolution: old wine in new skins. American Journal of Botany. 82:944-953.
(RHS) Colour Chart. 2007. Royal Horticultural Society, Londres (Gran Bretaña).

Seminario J., M. Valderrama, \& I. Manrique. 2003. El yacón: Fundamentos para el aprovechamiento de un recurso promisorio. Lima, Perú: Centro Internacional de la Papa (CIP), Universidad Nacional de Cajamarca y Agencia Suiza para el Desarrollo y la Cooperación (COSUDE).

Sidorov V.A., M.K. Zubko, A.A. Kuchko, I.K. Komarnitsky \& Y.Y. Gleba. 1987. Somatic hybridization in potato: use of y-irradiated protoplasts of Solanum pinnatisectum in genetic reconstruction. Theoretical and Applied Genetics. 74:364-368.

Singh R. 2012. Genetic resources, chromosome engineering, and crop improvement series: Medicinal Plants. 6 (20): 642-693.

Soto J. 2012. Evaluación de la diversidad genética de colecciones de Smallanthus sonchifolius (Poepp. \& Endl.) "Yacón" del Perú. Tesis, Magister en Mejoramiento Genético de Plantas. Universidad Nacional Agraria La Molina.

Svobodová E., Z. Dvoráková, P.H. Cepková, I. Viehmannová, L. Havlícková, E. Fernández, D. Russo \& G. Meza. 2009. Genetic diversity of yacón (Smallanthus sonchifolius (Poepp. \& Endl.) H. Robinson) and its wild relatives as revealed by ISSR Markers. Journal of Biochemical Systematics and Ecology. 50: 383-389.

UPOV. 2011. Documento conexo a la introducción general al examen de la distinción, la homogeneidad y la estabilidad y a la elaboración de descripciones armonizadas de las obtenciones vegetales. Documento TGP / 7. Elaboración de las Directrices de Examen. Ginebra, Suiza. 98 p. Consultado en línea http://www.upov.int/es/publications/tgp/documents/ tgp7_1.pdf.

Viehmannova I., Z. Bortlova, J. Vitamvas, P.H. Cepkova, K. Eliasova, E. Svobodova \& M. Travnickova. 2013. Assessment of somaclonal variation in somatic embryo-derived plants of yacón [Smallanthus sonchifolius (Poepp. and Endl.) H. Robinson] using inter simple sequence repeat analysis and flow cytometry. Electronic Journal of Biotechnology. 17:102-106. doi:10.1016/j.ejbt.2013.12.011. 
Anexo 1: Caracterización morfológica de las variedades de yacón empleadas como parentales para el Descriptor14: Color de la superficie de la raíz reservante, Descriptor 15: Color de la pulpa de la raíz reservante y Descriptor 16: Tendencia a formar hendiduras en las raíces reservantes.

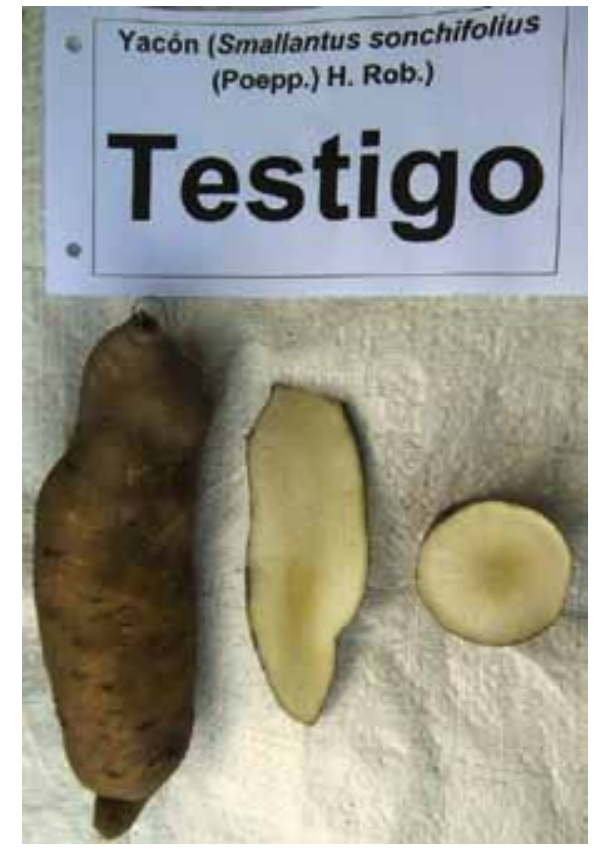

D14: Púrpura grisáceo claro

D15: Blanco

D16: Lisa

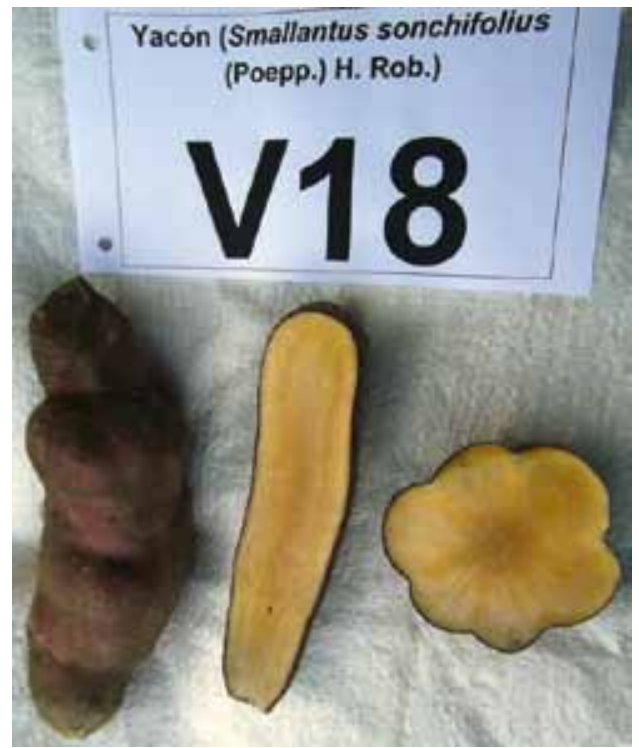

D14: Púrpura grisáceo claro

D15: Naranja claro

D16: Con hendiduras

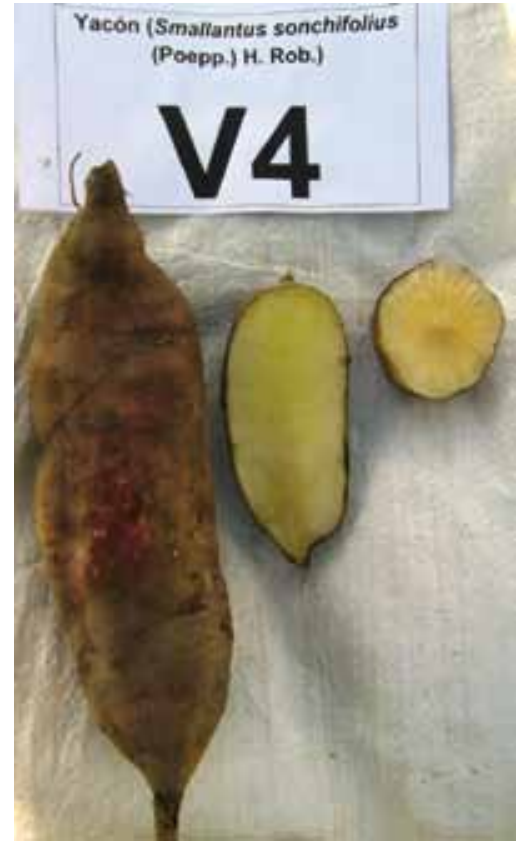

D14: Púrpura grisáceo oscuro

D15: Amarillo naranja

D16: Lisa

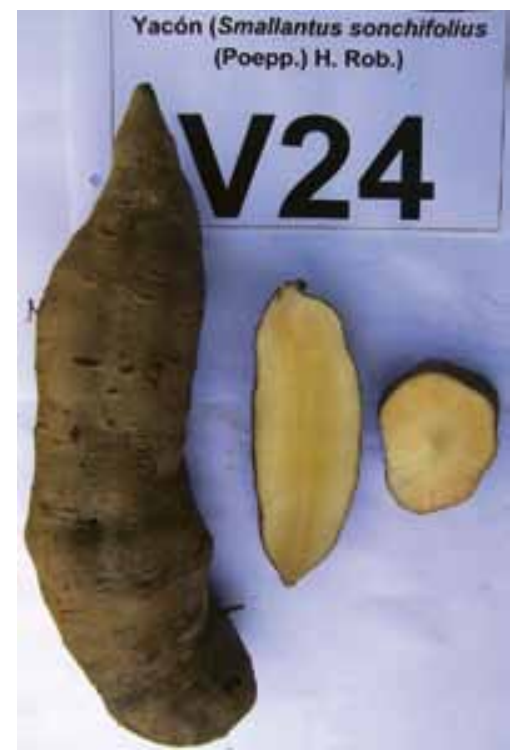

D14: Púrpura grisáceo oscuro

D15: Amarillo naranja

D16: Con hendiduras

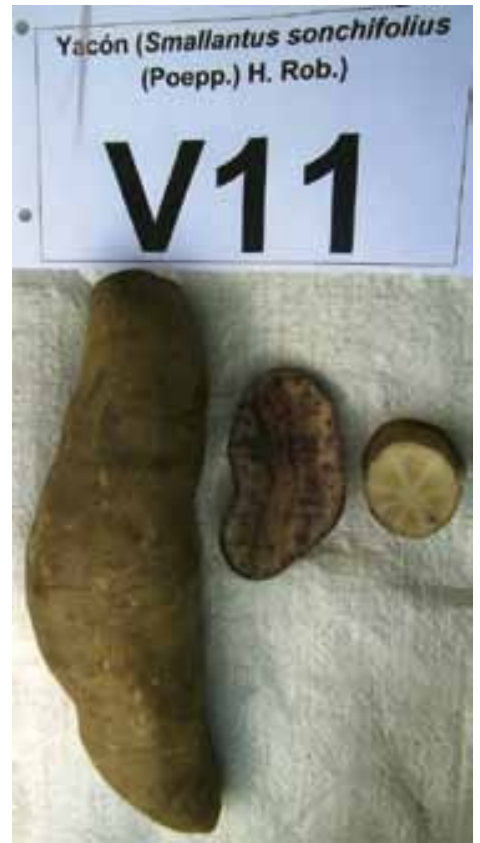

D14: Amarillo claro

D15: Blanco amarillento con motas irregulares púrpura rojizo

D16: Con hendiduras

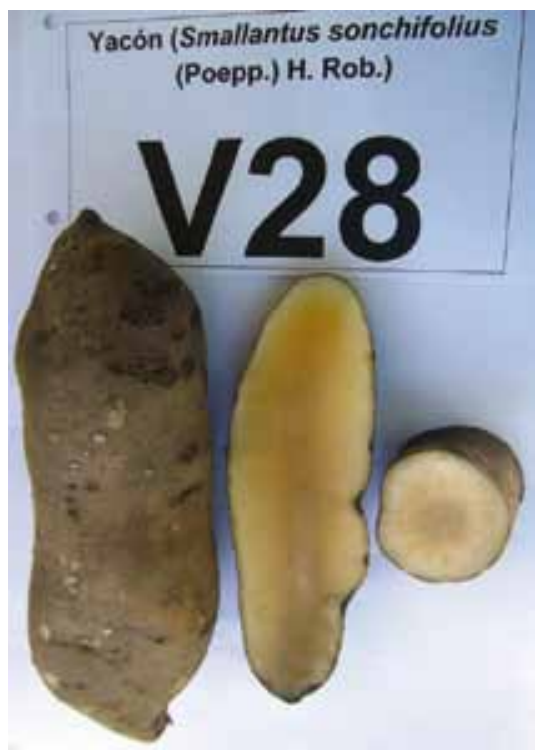

D14: Amarillo claro

D15: Amarillo naranja

D16: Lisa 
Anexo 2. Híbridos de yacón con status Positivo. Izquierda: Superficie de la raíz. Derecha: Corte longitudinal para la observación de la pulpa
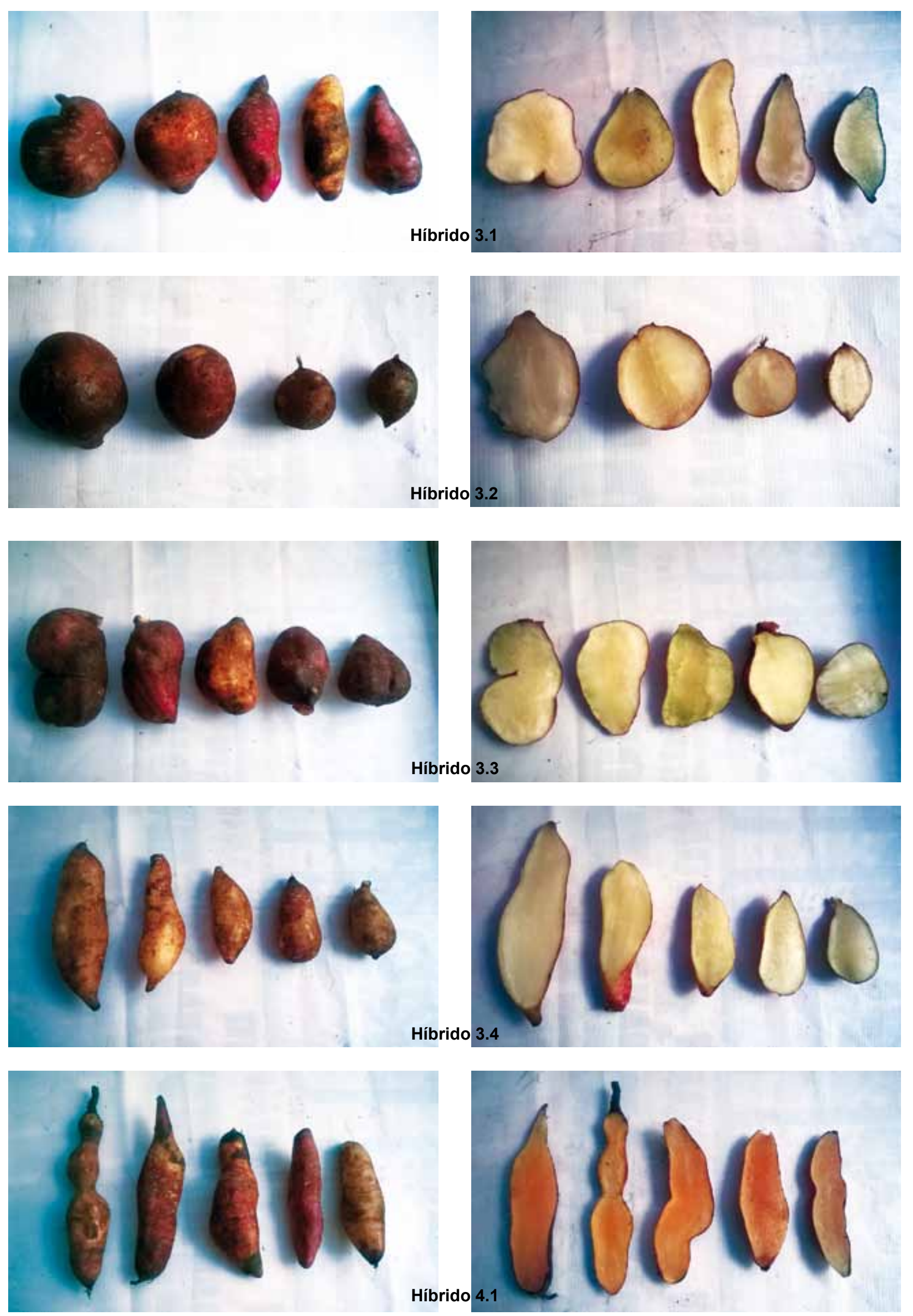


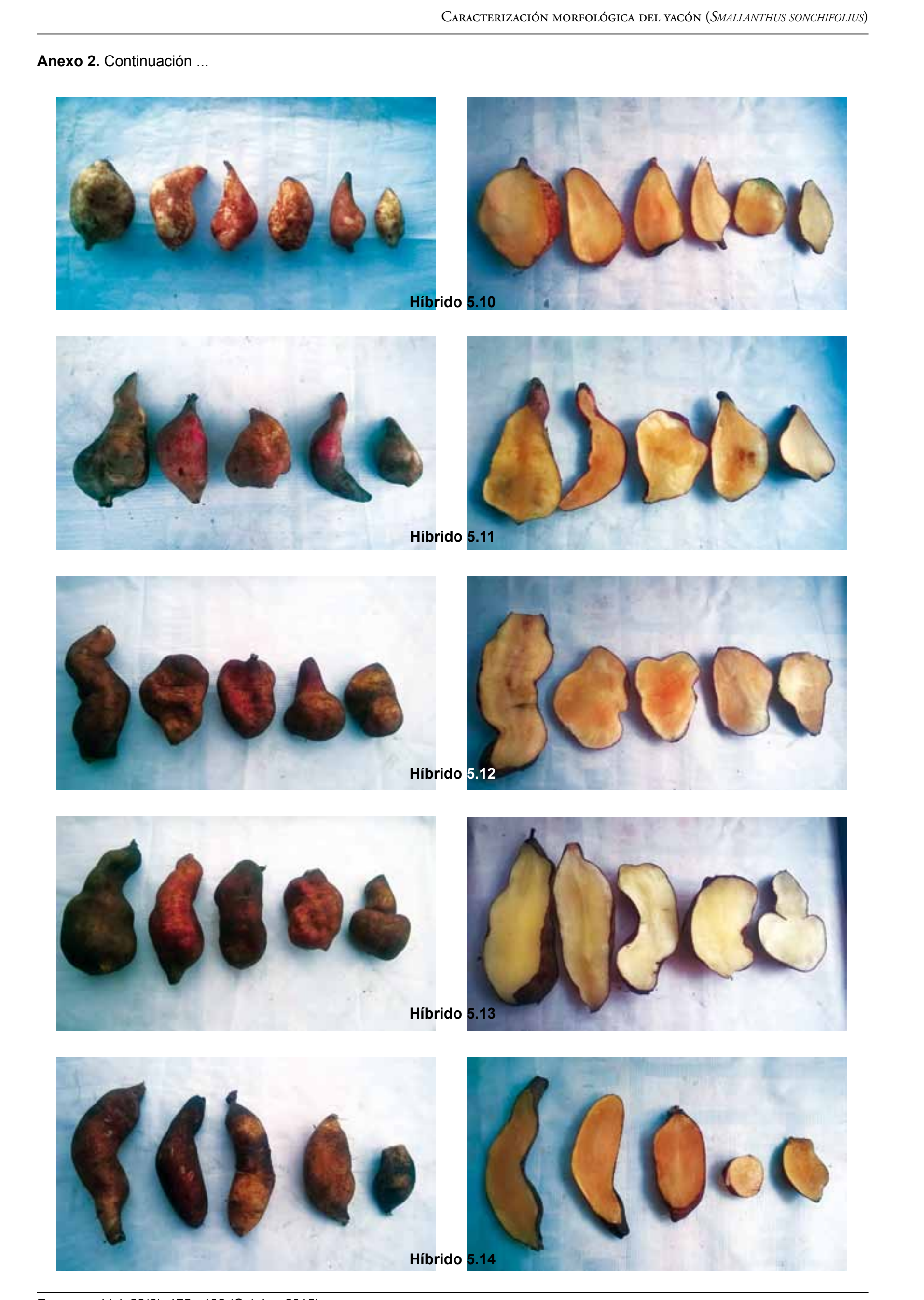




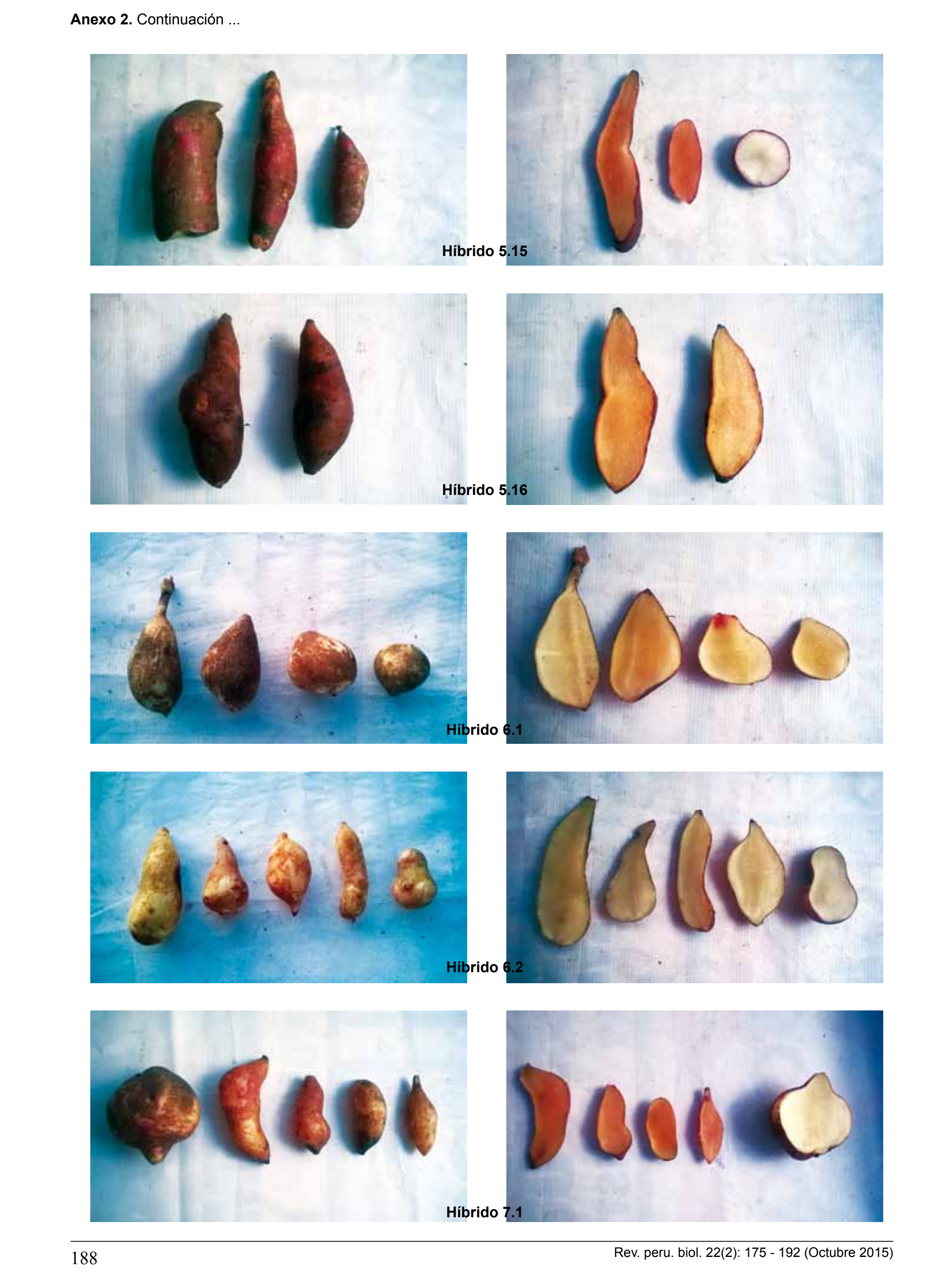




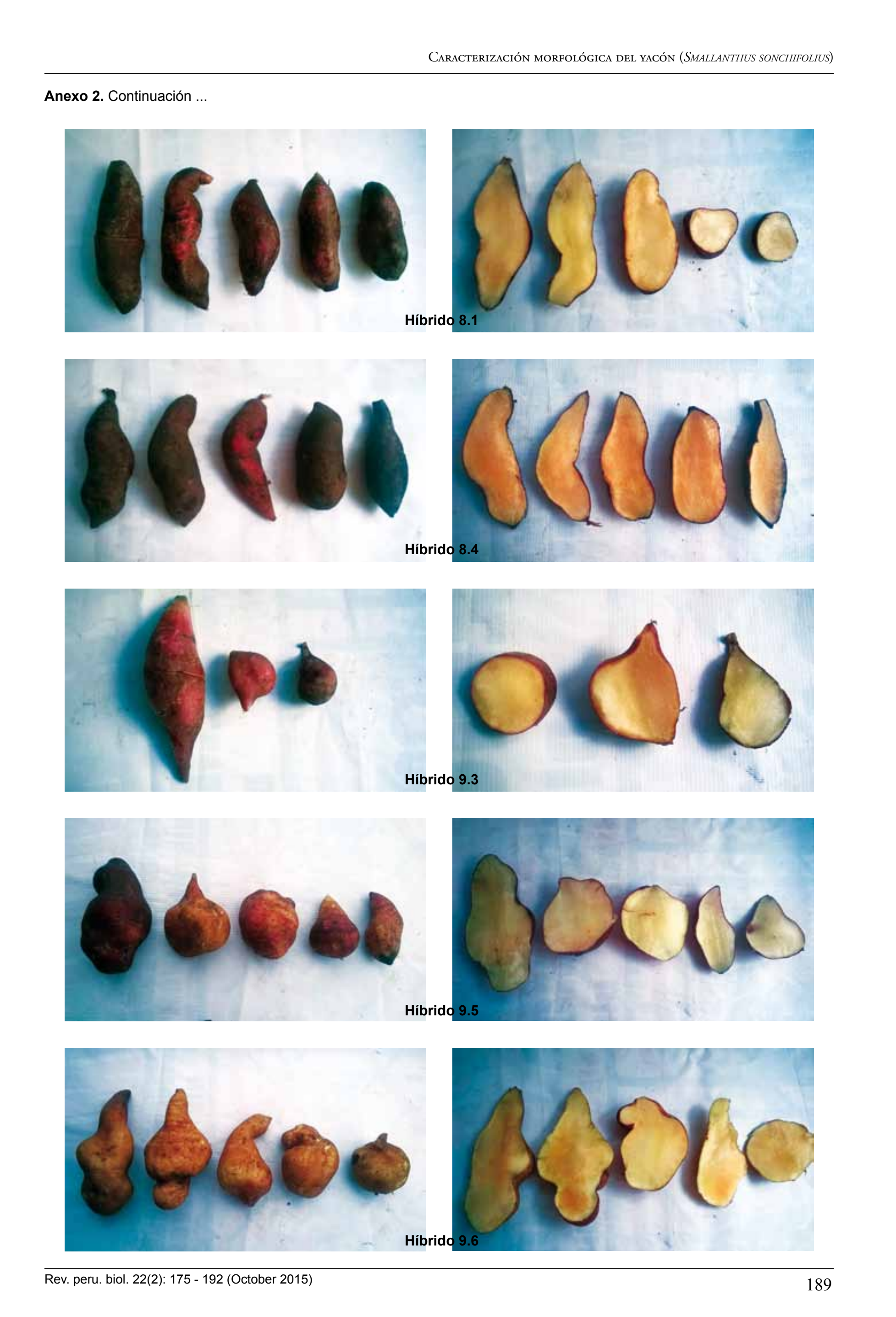




$$
\begin{aligned}
& \overline{\text { Jesue Dereo }} \\
& 100.000
\end{aligned}
$$

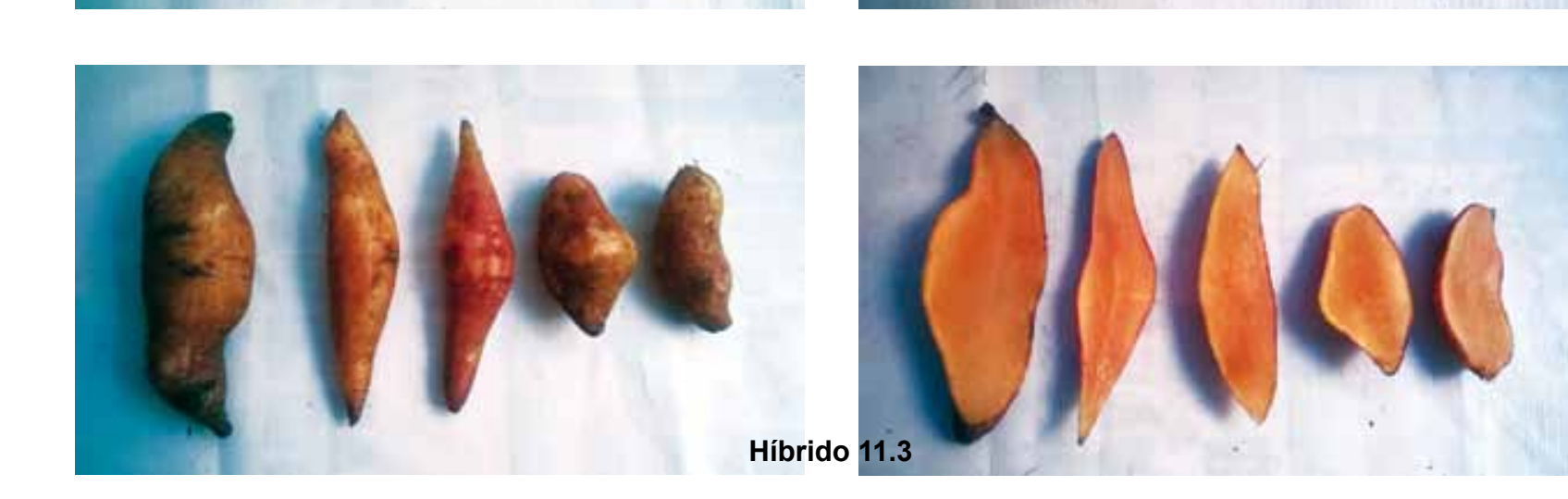

$$
\begin{aligned}
& \text { arsb Boce } \\
& \text { 6106. Drodo }
\end{aligned}
$$




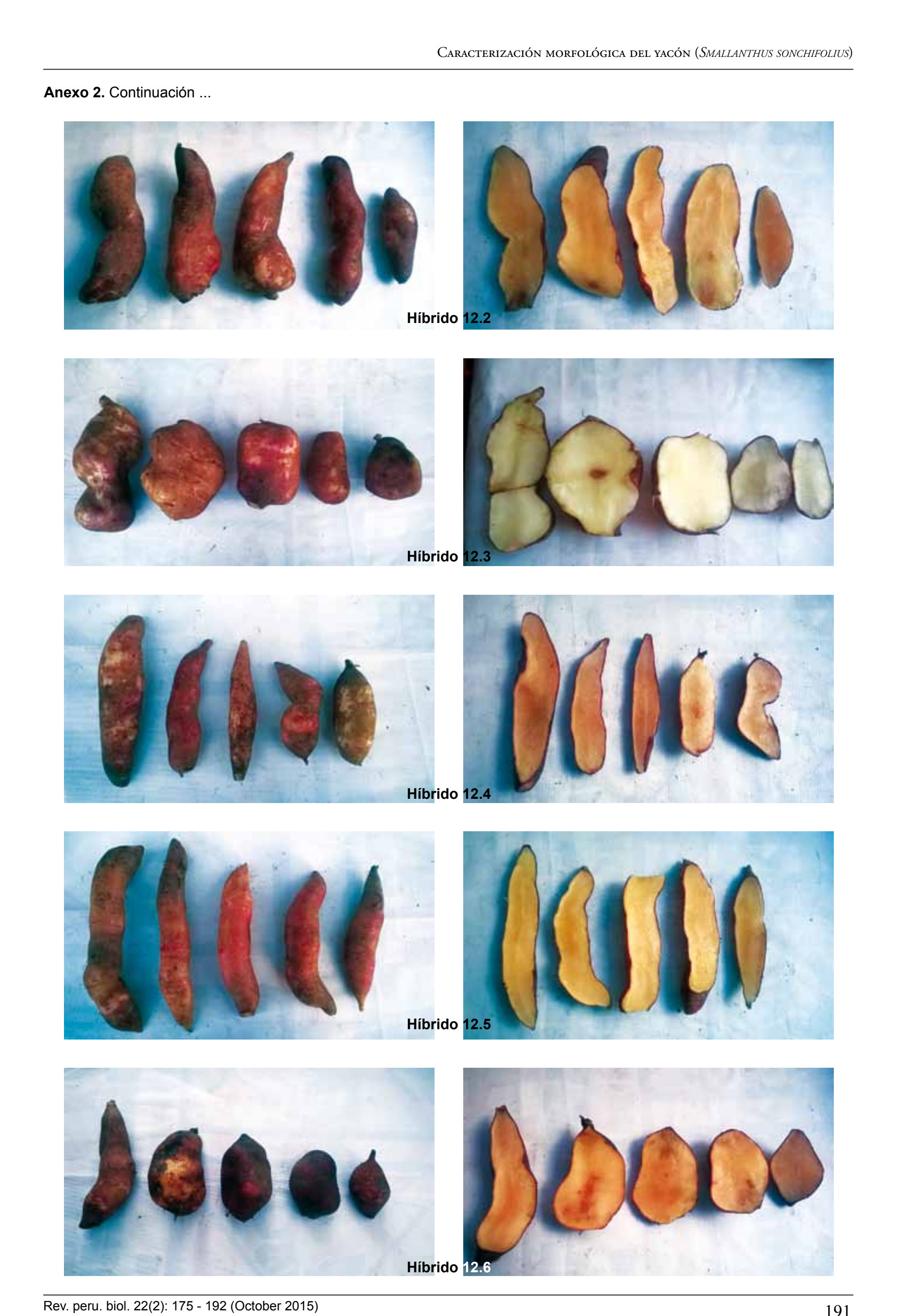




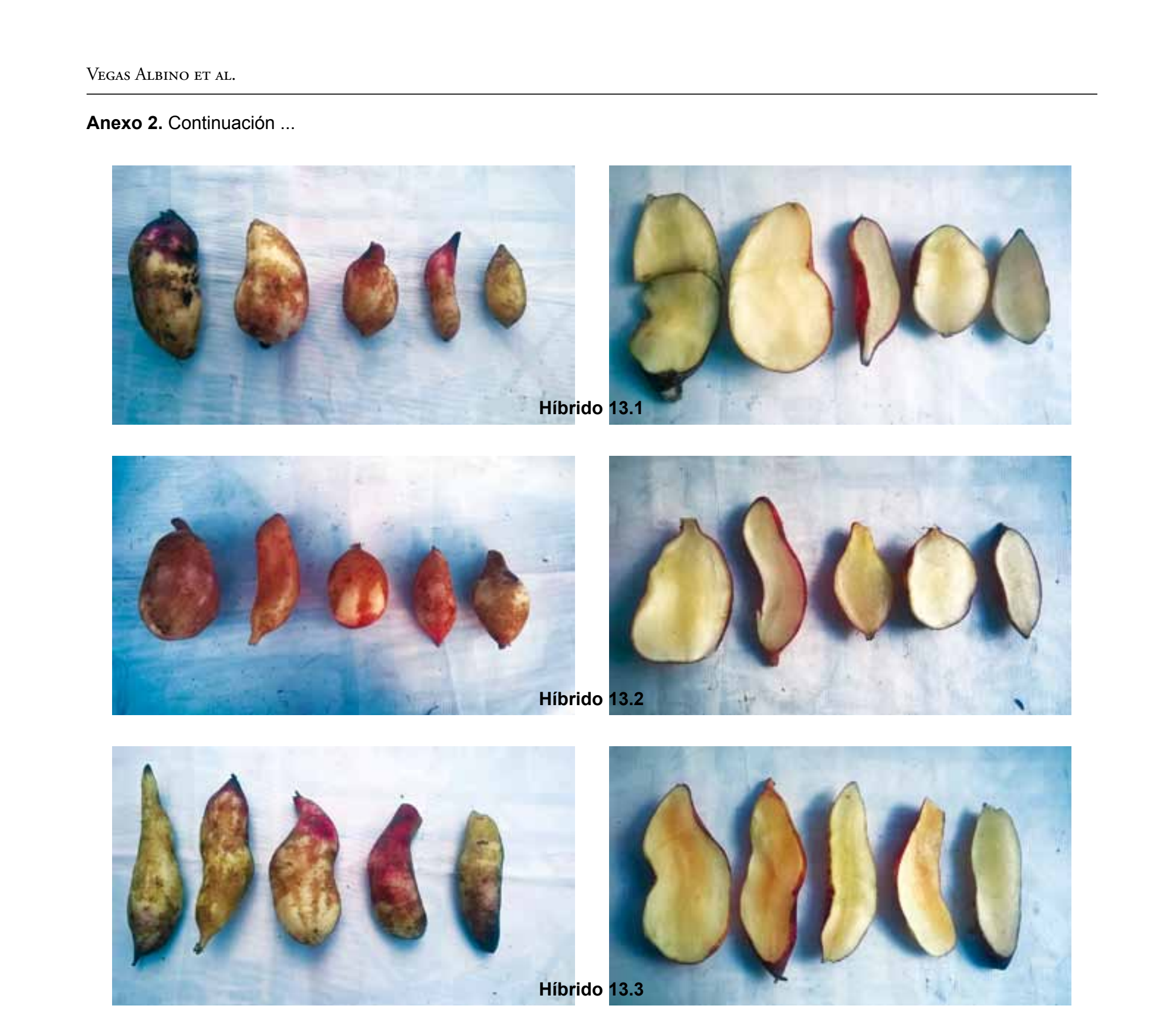

